\title{
Don't miss a chance taking the best shot!!
}

\author{
kunihiko kiuchi ${ }^{1}$ \\ ${ }^{1}$ Kobe University Graduate School of Medicine
}

September 21, 2020

\section{RESPONSE FOR THE LETTER TO THE EDITOR}

Don't miss a chance taking the best shot!!

Kunihiko Kiuchi

Division of Cardiovascular Medicine, Department of Internal Medicine, Kobe University Graduate School of Medicine

Address for correspondence:

Kunihiko Kiuchi, MD, FHRS

Section of Arrhythmia, Division of Cardiovascular Medicine,

Department of Internal Medicine, Kobe University Graduate School of Medicine

7-5-2 Kusunoki-chou chuou-ku, Kobe, Hyogo, Japan

Telephone: (81)-78-382-5846

Fax: (81)-78-382-5859

E-mail: kunihikokiuchi@yahoo.co.jp

Disclosures:

The Section of Arrhythmia was supported by an endowment from Medtronic JAPAN and Abbott JAPAN. The authors have reported that they have no relationships relevant to the contents of this paper to disclose.

Funding: None

To the Editor,

We thank Dr. Althoff and Mont for their interest, comments, and suggestions related to our paper. ${ }^{1}$ In our study, as they suggested, LGE-MRI was acquired 1-3 months after the ablation. An animal study reported that ablation lesions are fully formed by 3 weeks and are dense with collagen and fat deposition by 6 weeks. $^{2}$ In our pilot study, the ablation lesion area dramatically diminished from 1 week to 6 weeks after the procedure in most of the patients. Furthermore, the ablation lesion area was comparable between 6 weeks and just 3 months after the ablation. Of interest, the ablation lesion disappeared after 5-6 months following the procedure in some patients without AF recurrence. We speculated that the shrinkage of the ablation lesion might have occurred during the pathophysiological healing process and reverse remodeling of the left atrium. Therefore, we considered that the time point of taking the "best shot" might have been earlier than we expected. Furthermore, the dose of the contrast agent was important for determining the time point. Our dose of the contrast agent was $0.1 \mathrm{mmol} / \mathrm{kg}$, which was relatively lower than that of the other groups. To address this issue, further study will be needed. 
As for an internal reference for normalization, the threshold of the signal intensity was initially determined according to the signal intensity histogram on the "whole LA wall" in our previous studies. ${ }^{3,4}$ However, as the authors suggested, neither the ablation lesion nor atrial fibrosis could be accurately visualized in some patients with advanced atrial remodeling. Therefore, the internal reference was changed from the "whole LA wall" to the "healthy LA wall" in our recent studies. ${ }^{1,5}$ We believed that this simple but important tip might make it possible to sensitively identify pre-existing atrial fibrosis, particularly interstitial fibrosis. The signal intensity of ablation lesion was significantly higher than that of the pre-existing atrial fibrosis. Our question is whether the ablation lesion characteristics dramatically differed between the different references in patients without atrial remodeling. Although we re-analyzed the LGE-MRI with two different references ("whole LA wall" and "healthy LA wall"), no significant difference in the lesion characteristics could be found. Cryoballoon ablation and RF ablation with contact-force sensing catheter induced intensive inflammation which followed by the artificial fibrosis. ${ }^{6}$ In this study, we focused on the visualization of the ablation lesion not the pre-existing atrial fibrosis, and patients without atrial remodeling were enrolled. We considered that the impact of the difference in the reference could be less than we expected, when we focused on the ablation lesions in patients without atrial remodeling.

To visualize the atrial fibrosis and the ablation lesion, different visualization methods were developed. However, very few histological validations could be found. Furthermore, the dose of the contrast agents, MRI system, and visualization software completely differed due to the regulations in each institution. Therefore, it is not surprising that the image differed between each visualization technique. It is important to note the reproducibility of the visualization method and clinical implication of the best shot. The authors had already reported the reproducibility of their method. ${ }^{7}$ However, this issue is still in debate. ${ }^{8}$ We would like to challenge to improve the quality of our "best shot" and strengthen both reproducibility and clinical implication in a further study.

\section{References}

1. Kurose J, Kiuchi K, Fukuzawa K, et al. Lesion characteristics between cryoballoon ablation and radiofrequency ablation with a contact-force sensing catheter: late-gadolinium enhancement magnetic resonance imaging assessment. J Cardiovasc Electrophysiol. 2020.

2. Avitall B, Kalinski A. Cryotherapy of cardiac arrhythmia: From basic science to the bedside. Heart Rhythm. 2015;12(10):2195-2203.

3. Shigenaga Y, Kiuchi K, Ikeuchi K, et al. Fusion of Delayed-enhancement MR Imaging and Contrastenhanced MR Angiography to Visualize Radiofrequency Ablation Scar on the Pulmonary Vein. Magn Reson Med Sci. 2015;14(4):367-372.

4. Kiuchi K, Okajima K, Shimane A, et al. Visualization of the radiofrequency lesion after pulmonary vein isolation using delayed enhancement magnetic resonance imaging fused with magnetic resonance angiography. J Arrhythm. 2015;31(3):152-158.

5. Akita T, Kiuchi K, Fukuzawa K, et al. Lesion distribution after cryoballoon ablation and hotballoon ablation: Late-gadolinium enhancement magnetic resonance imaging analysis. J Cardiovasc Electrophysiol. 2019 .

6. Kiuchi K, Fukuzawa K, Mori S, Watanabe Y, Hirata KI. Feasibility of Imaging Inflammation in the Left Atrium Post AF Ablation Using PET Technology. JACC Clin Electrophysiol. 2017;3(12):1466-1467.

7. Benito EM, Carlosena-Remirez A, Guasch E, et al. Left atrial fibrosis quantification by late gadoliniumenhanced magnetic resonance: a new method to standardize the thresholds for reproducibility.Europace. 2017;19(8):1272-1279.

8. Kamali R, Schroeder J, DiBella E, et al. Reproducibility of Clinical Late Gadolinium Enhancement Magnetic Resonance Imaging in Detecting Left Atrial Scar after Atrial Fibrillation Ablation. J Cardiovasc Electrophysiol. 2020 in press. 